\title{
Teores foliares e esclerofilia de Calophyllum brasiliense Cambess. (Clusiaceae) e suas relações edáficas no litoral do Estado do Paraná, Brasil ${ }^{1}$
}

\author{
Fernanda Sereda ${ }^{2}$, Carlos Bruno Reissmann², Maria Regina Boeger ${ }^{3,4}$ e Renato Marques $^{2}$
}

Recebido: 3.12.2015; aceito: 30.09.2016

\begin{abstract}
Foliar content and esclerophylly of Calophyllum brasiliense Cambess. (Clusiaceae) and their edaphic relationships in coast of Paraná State, Brazil). This study evaluated the relationship between soil nutrient content and foliar nutrient content of Calophyllum brasiliense in two sites from the coast of Paraná State, with different fertility conditions. The soils were analysed for chemical attributes and leaves for nutrients, $\mathrm{Al}, \mathrm{Na}$ and specific leaf area as sclerophylly index. Leaves showed differences only for $\mathrm{K}, \mathrm{Ca}, \mathrm{Cu}$ contents and specific leaf area. The $\mathrm{K}$ and $\mathrm{Ca}$ contents were higher in the leaves of trees from Rio Velho, while the $\mathrm{Cu}$ content and specific leaf area were higher in the leaves of trees from Floresta do Palmito. $\mathrm{Al}$ and $\mathrm{Na}$ contents in leaves indicated that this species is tolerant to these elements. The specific leaf area indicated that the leaves are sclerophyllous in response to stress conditions due to low nitrogen content in both sites, besides the high $\mathrm{Al}$ content and low soil fertility in Floresta do Palmito and high soil salinity in Rio Velho.
\end{abstract}

Keywords: guanandi, leaf nutrition, lowland tropical forest, soil fertility

RESUMO - (Teores foliares e esclerofilia de Calophyllum brasiliense Cambess. (Clusiaceae) e suas relações edáficas no litoral do Estado do Paraná, Brasil). Este estudo avaliou a relação entre teores de nutrientes do solo e foliares de Calophyllum brasiliense (guanandi), em dois sítios do litoral paranaense, em distintas condições de fertilidade. Os atributos químicos do solo e foliares, Al, Na e área específica foliar, como índice de esclerofilia, foram analisados. As folhas diferiram para os teores de $\mathrm{K}, \mathrm{Ca}$ e $\mathrm{Cu}$ e área específica foliar. Os teores de $\mathrm{K}$ e $\mathrm{Ca}$ foram maiores nas folhas das plantas do Rio Velho, enquanto que os teores de $\mathrm{Cu}$ e a área específica foliar foram maiores nas folhas das plantas da Floresta do Palmito. Os teores de $\mathrm{Al}$ e Na indicaram que a espécie é tolerante a esses elementos. A área foliar específica indicou que as folhas são esclerófilas como resposta à condição de estresse devido ao baixo teor de $\mathrm{N}$ em ambos os sítios, além do alto teor de $\mathrm{Al}$ e a baixa fertilidade do solo na Floresta do Palmito e à alta salinidade do solo no Rio Velho.

Palavra-chave: guanandi, fertilidade do solo, floresta ombrófila densa de terras baixas, nutrição foliar

\section{Introdução}

A distribuição das plantas nos ecossistemas florestais é influenciada pelas características do sítio onde ocorrem, sendo o solo é um dos principais modificadores dessa distribuição. Por outro lado, a vegetação também influencia nas propriedades do solo. As trocas químicas entre o solo e as plantas por meio de absorção, distribuição dentro da planta e lixiviação de nutrientes, além da desfolhação e decomposição foliar, compõem os principais mecanismos da ciclagem de nutrientes florestais (Prescott 2002).
Assim, a análise nutricional foliar permite caracterizar as espécies vegetais e estabelecer uma relação entre os teores de nutrientes no solovegetação. Essas correlações nem sempre são diretas, porque as espécies vegetais diferem quanto às suas exigências nutricionais no tempo e no espaço. Em sistemas naturais, as exigências nutricionais de cada espécie respondem à uma complexa interação entre as variáveis ambientais que atuam no processo de ciclagem de nutrientes (Lambers et al. 1998).

Em ambientes de baixa fertilidade, a aquisição de nutrientes do solo ocorre pela otimização da

1. Parte da Dissertação de Mestrado da primeira Autora

2. Universidade Federal do Paraná, Departamento de Solos e Engenharia Agrícola Rua dos Funcionários, 1540, Bairro Juvevê, 80.035050, Curitiba, PR

3. Universidade Federal do Paraná, Departamento de Botânica, Caixa postal 19031, Centro Politécnico, Bairro Jardim das Américas, 81531-980 Curitiba, PR

4. Autor para correspondência: mrtboeger@gmail.com 
ciclagem de nutrientes, onde o input de nutrientes ocorre pela incorporação da biomassa endógena no solo (Protil et al. 2009), devido a decomposição da serapilheira (Ndaw et al. 2009). Muitas espécies vegetais apresentam adaptações como medida preventiva para evitar perda de nutrientes por meio de ajustes morfológicos, desenvolvendo barreiras físicas (ex. escleromorfismo), ajustes fisiológicos (ex. translocação de nutrientes) e químicos (ex. compostos fenólicos) (Hanley et al. 2007).

O Estado do Paraná apresenta atualmente menos de 5\% da cobertura original (Santo Jr. 2008). Desta cobertura, destaca-se a Floresta Atlântica que se diferencia das demais formações florestais pela heterogeneidade, tanto na sua origem quanto na sua biodiversidade. Na planície litorânea, esse ecossistema é considerado frágil pois, na sua maioria, está sobre solos inférteis e lixiviados. A fertilidade das áreas está restrita aos primeiros $10 \mathrm{~cm}$ do solo, onde se encontra de 70 a $80 \%$ do sistema radicial das espécies vegetais (Bonilha 2012).

Entre as muitas espécies que ocupam a planície litorânea no sul do Brasil, destaca-se o Calophyllum brasiliense Camb. (Clusiaceae), conhecido como guanandi. A espécie é classificada tanto como intermediária tardia ou clímax, como pioneira nas formações de influência fluvial, formando os guanandizais (Carvalho 2003). Em estudos fitossociológicos, o guanandi apresenta altos valores de importância, principalmente em florestas com solos hidromórficos (Zacarias et al. 2012).

Devido à dominância desta espécie em diferentes formações florestais no litoral paranaense, este estudo relacionou os nutrientes do solo, em dois sítios com diferentes condições de fertilidade, com os teores foliares de Calophyllum brasiliense.

\section{Material e métodos}

O estudo foi conduzido em dois sítios: 1) Rio Velho $\left(25^{\circ} 14^{\prime} \mathrm{S}\right.$ e $\left.48^{\circ} 26^{\prime} \mathrm{W}\right)$ localizado na Reserva Natural Serra do Itaqui, pertencente à Sociedade de Pesquisa em Vida Selvagem e Educação Ambiental (SPVS), Guaraqueçaba, PR. O sítio apresenta Neossolo Flúvico Sódico Salino sob influência do clima Af (t). 2) Floresta Estadual do Palmito (253' S e 48 $32^{\prime} \mathrm{W}$ Gr), unidade de conservação administrada pelo Instituto Ambiental do Paraná (IAP), Paranaguá, PR. Esta floresta está sob a influência do clima tipo Af e apresenta solo do tipo Espodossolo Cárbico Órtico.

Para cada sítio, foram selecionadas seis árvores e amostras de solo foram coletadas nas profundidades de 0-5; 5-10 e 10-20 cm, na projeção da copa de cada árvore selecionada. As amostras de solo foram obtidas por meio da média das três profundidades amostradas. O solo foi seco ao ar natural e peneirado. Foram determinados o $\mathrm{pH}$ em $\mathrm{CaCl}_{2} 0,01 \mathrm{molL}^{-1}, \mathrm{H}^{+1}$ $+\mathrm{Al}^{+3}$ (obtido com a solução SMP), Alumínio (Al), Cálcio $(\mathrm{Ca})$ e Magnésio $(\mathrm{Mg})$ trocáveis (extraídos com solução $\mathrm{KCl} 1 \mathrm{molL}^{-1}$ e determinados por titulação), Fósforo (P), Potássio (K) e Sódio (Na) trocáveis (extraídos com Mehlich 1 e determinados por colorimetria com azul de molibdênio e fotometria de chama para $\mathrm{K}$ e $\mathrm{Na}$ ) e Carbono (C) orgânico por colorimetria (Pavan 1992). Com base nestes dados, foram calculadas a capacidade de troca de cátions (CTC) efetiva e a porcentagem de saturação por bases (V\%). O sódio foi assim calculado: $\mathrm{Na} \%=100$ $\times \mathrm{Na}$ /CTC. O Nitrogênio $(\mathrm{N})$ total foi determinado pelo processo micro Kjeldahl (Bremmer 1996). Ferro (Fe), Manganês ( $\mathrm{Mn})$, Zinco $(\mathrm{Zn})$ e Cobre $(\mathrm{Cu})$ foram extraídos em solução $\mathrm{HCl} 0,1 \mathrm{molL}^{-1}$ e determinados por absorção atômica (Silva 1999). A condutividade elétrica (CE) foi determinada pelo extrato aquoso 1:2 (Raij et al. 2001).

A coleta das folhas verdes e totalmente expandidas foi realizada na face norte e no terço inferior da copa das árvores. As folhas foram lavadas com água deionizada e secas em estufa à $60{ }^{\circ} \mathrm{C}$ com ventilação forçada. Após a secagem, o material foi moído e homogeneizado. O mesmo procedimento foi aplicado para as seis amostras da serapilheira das folhas de guanandi coletadas próximas aos indivíduos marcados, nos dois sítios. Os teores dos macro e micronutrientes, Al e $\mathrm{Na}$ nas folhas verdes e na serapilheira acumulada foram determinados de acordo com Martins \& Reissmann (2007). Os teores de C e Nitrogênio (N) foram determinados por combustão, em analisador CNHS Elementar (Mod.Vario-El).

A área específica foliar $\left(\mathrm{AEF}, \mathrm{cm}^{2} \mathrm{~g}^{-1}\right)$ foi calculada a partir da razão área foliar $\left(\mathrm{cm}^{2}\right) /$ massa foliar $(\mathrm{g})$ para dez folhas por indivíduo/sítio. As folhas, desidratadas à $60{ }^{\circ} \mathrm{C}$, foram pesadas em balança de precisão. Nas folhas, a área foliar foi estimada a partir da imagem digitalizada em "scanner" de mesa com o programa SIGMASCAN-PRO (versão 5.0, SPSS In., Chicago Il, USA). O material vegetal de referência (UPCB 44428) está depositado no Herbário do Departamento de Botânica, UFPR.

As variáveis do solo e foliares foram submetidas à análise de variância e o teste de médias de Duncan $(p<0,05)$ para comparação dos dois sítios, no 
programa STATISTICA 6.0 (StatSoft, INc., Tulsa, OK, USA).

\section{Resultados e Discussão}

As médias dos atributos químicos do solo apresentaram diferenças entre os sítios estudados, com exceção do $\mathrm{H}+\mathrm{Al}$ (tabela 1). Os valores médios de $\mathrm{H}+\mathrm{Al}$, estimados pela solução SMP, representam o $\mathrm{H}$ e $\mathrm{Al}$ adsorvidos às cargas negativas dos argilominerais, óxidos de $\mathrm{Fe}$ e $\mathrm{Al}$ e grupos funcionais do húmus capazes de dissociarem $\mathrm{H}^{+}$. Essa equivalência nos valores da acidez potencial para solos tão distintos quanto aos deste estudo, pode ser explicada em função da CTC, matéria orgânica (MO), $\mathrm{pH}$ do solo, teor e tipo de argila, atuando de forma distinta sobre a solução de equilíbrio SMP (Raij 1991, Silva et al. 2006). No Rio Velho, a condição de alagamento representa um fator adicional pela redução promovendo o consumo de $\mathrm{H}$ (Meurer 2006). Especificamente na Floresta do Palmito, com predominância da textura arenosa, pode-se atribuir à menor quantidade de $\mathrm{MO}$ a maior expressão no suprimento da acidez potencial, enquanto que no Rio Velho esta fonte deve advir da maior contribuição dos argilominerais e óxidos, além da MO.

Apesar dessas características, ambos os sítios são extremamente ácidos. As médias dos atributos químicos do solo apresentaram diferenças entre os sítios estudados, com exceção do $\mathrm{H}+\mathrm{Al}$ (tabela 1). Os valores médios de $\mathrm{H}+\mathrm{Al}$ (índice SMP) foram similares devido à condição de hidromorfia desses sítios e pela baixa CTC (Meurer 2006), resultante da textura arenosa (tabela 1).

Em relação ao Al, os sítios são distintos, uma vez que a Floresta do Palmito é $280 \times$ mais saturada em Al do que o Rio Velho, com base na CTC (tabela 1). Essa diferença é marcante e influencia a acidez do solo e, consequentemente, a disponibilidade de outros elementos como o $\mathrm{P}, \mathrm{Ca}$ e $\mathrm{Cu}$ (George \& Neumann 2012). Nas quantidades em que se encontra no solo, o Al pode ser considerado tóxico para as plantas (Geoghegan \& Sprent 1996).

Os valores médios de $\mathrm{Ca}, \mathrm{Mg}, \mathrm{K}, \mathrm{Na}, \mathrm{P}, \mathrm{C}, \mathrm{N}$ e condutividade elétrica foram superiores no solo do Rio Velho em relação ao solo da Floresta do Palmito. A condição de redução, no Rio Velho, proporciona o aumento no $\mathrm{pH}$ do meio e a solubilização de outros cátions é favorecida (Meurer 2006). Pelo fato do Rio Velho sofrer influência fluviomarinha, os valores médios da CE permitem classificá-lo como salinosódico (EMBRAPA 1999).
Os maiores valores médios de $\mathrm{C}$ e $\mathrm{N}$ no solo do Rio Velho são semelhantes aos valores encontrados no levantamento realizado pela SPVS (2002), onde os Neossolos da região de Guaraqueçaba apresentaram altos valores de $\mathrm{C}$, devido as adições de $\mathrm{MO}$ provenientes da deposição dos materiais aluviais.

Porém, a maior relação C: $\mathrm{N}$ encontrada foi no solo da Floresta do Palmito, devido aos baixos teores de $\mathrm{N}$. A relação $\mathrm{C} / \mathrm{N}>30$ indica o esgotamento do $\mathrm{N}$ no solo, o que torna esse elemento restrito para as plantas (Luchese et al. 2001).

Com relação aos micronutrientes, os maiores valores médios foram encontrados no solo Rio Velho (tabela 1). A forte gleização no solo promove a redução do Fe, uma vez que nesse sítio é comum a deficiência na drenagem (SPVS 2002). É importante destacar que os teores de $\mathrm{Fe}, \mathrm{Mn}, \mathrm{Zn}$ e $\mathrm{Cu}$ são considerados altos no Rio Velho enquanto que os teores de $\mathrm{Zn}, \mathrm{Mn}$ e $\mathrm{Cu}$ variam de médios a baixos na Floresta do Palmito. A variação dos teores parece ter relação com o material de origem, a dinâmica local e a reciclagem proporcionada pela matéria orgânica acumulada na superfície (Barber 1995, SPVS 2002). A MO, juntamente com o pH, tem uma influência no comportamento dos micronutrientes no solo, particularmente o $\mathrm{Cu}$. A adsorção do $\mathrm{Cu}$ é fortemente influenciada pela $\mathrm{MO}$, tanto na superfície dos agregados como entre os agregados (Rieuwerts 2007).

Com base nos valores médios da CTC efetiva e V\% (tabela 1), o solo do sítio Rio Velho apresentou maior fertilidade em relação ao sítio Floresta do Palmito. A CTC na Floresta do Palmito é baixa (1\%), ao contrário do Rio Velho. Essa fertilidade poderia favorecer o crescimento do guanandi; entretanto, o solo do Rio Velho apresentou alto teor de $\mathrm{Na}$. O Na contribuiu em $40,70 \%$ na CTC e nos maiores valores da condutividade elétrica (CE), indicando que somente espécies tolerantes à salinidade podem se desenvolver nessa condição (Tomé Júnior 1997). Com base nos valores da CE do Rio Velho $(9,9 \pm 2,3)$, calculou-se o valor da porcentagem de saturação do Na trocável na CTC (PST $=40,7 \pm 5,1)$. Juntamente com os valores de pH e CE (tabela 1), o solo do Rio Velho foi classificado como salino-sódico (Meurer 2006).

Os teores de $\mathrm{K}$, $\mathrm{Ca}$ e $\mathrm{Cu}$ apresentaram diferenças significativas entre os as folhas dos indivíduos dos sítios estudados (tabela 2), apesar da diferença dos teores de nutrientes do solo. Os valores médios de $\mathrm{K}$ e Ca foram maiores no Rio Velho, enquanto que o $\mathrm{Cu}$ foi maior na Floresta do Palmito. Os teores médios dos macronutrientes e $\mathrm{Na}$ foliares apresentaram a seguinte 
ordem: $\mathrm{N}>\mathrm{K}>\mathrm{Ca}>\mathrm{Mg}>\mathrm{P}>$ Na para o Rio Velho e $\mathrm{N}>\mathrm{K}>\mathrm{Mg}>\mathrm{Ca}>\mathrm{P}>$ Na para o Floresta do Palmito (tabela 2).

Os baixos valores médios de Ca nos indivíduos da Floresta do Palmito podem ser justificáveis pela elevada acidez desse local, pelos baixos teores disponíveis no solo ou pela afinidade dos sítios de troca da fração orgânica por esse elemento (Barber 1995). Adicionalmente, formas não trocáveis de Ca podem não ter sido detectadas pelos métodos de rotina e representar uma grande parte do total desse elemento disponível para plantas.

Os valores médios de $\mathrm{K}$ foram menores nos indivíduos na Floresta do Palmito. Devido à alta mobilidade desse elemento, tanto no solo quanto nas plantas (Lambers 1998), fatores como a alta pluviosidade podem favorecer sua lixiviação. Além disso, os baixos valores encontrados nos indivíduos da Floresta do Palmito pode ter ocorrido pela translocação desse elemento, devido a sua alta mobilidade (Protil et al. 2006).

A concentração média dos micronutrientes e Al nas folhas de guanandi deu-se da seguinte maneira: $\mathrm{Al}>\mathrm{Fe}$
$>\mathrm{Mn}>\mathrm{Zn}>\mathrm{Cu}$, para ambos os sítios (tabela 2). Com exceção do $\mathrm{Cu}$, cuja concentração foi maior nas folhas das plantas da Floresta do Palmito, as concentrações de micronutrientes foram semelhantes entre os dois sítios. A concentração de $\mathrm{Cu}$ foi maior no solo do Rio Velho e, portanto, inversamente proporcional a concentração das folhas. Em solos arenosos ricos em MO, como os da Floresta do Palmito, determinadas formas orgânicas são mais aptas a tornar o $\mathrm{Cu}$ indisponível, uma vez que complexos de $\mathrm{Cu}$ de baixo peso molecular $(\leq 1000)$, tendem a liberar $\mathrm{Cu}$ para as plantas enquanto que, substâncias húmicas de peso molecular $\geq 5000$ dificultam sua absorção (Mengel \& Kirkby 1987).

Em solos com baixa relação $\mathrm{C} / \mathrm{N}$, como ocorre no Rio Velho $(8,75)$, a decomposição é mais rápida, o que pode influenciar a velocidade de liberação desse elemento para a vegetação (Jordão et al. 2000). Na Floresta do Palmito, a C/N é muito maior $(58,7)$, o que torna a decomposição mais lenta. Além disso, deve-se considerar também a acidez dos sítios de coleta, ambos ácidos, que também interferem no processo de adsorção do $\mathrm{Cu}$ (Silveira \& Alleoni 2003).

Os teores de Al nas folhas dos indivíduos do Rio Velho e da Floresta do Palmito são semelhantes entre

Tabela 1. Médias e respectivos desvios-padrão dos atributos químicos, condutividade elétrica (CE), alumínio (Al) da camada de $0-20 \mathrm{~cm}$ do solo nos sítios de coleta. Letras diferentes em linha representam diferenças significativas, teste de Duncan, $\alpha=5 \%$.

Table 1. Mean values and respective standard deviations of chemical traits, electrical conductivity (CE), aluminum (Al) from $0-20 \mathrm{~cm}$ of soil layer in the studied sites. Different letters on the line represent significant differences, Duncan test, $\alpha=5 \%$.

\begin{tabular}{lcc}
\hline \multirow{2}{*}{ Atributos } & \multicolumn{2}{c}{ Sítios } \\
\cline { 2 - 3 } & Rio Velho & Floresta do Palmito \\
\hline $\mathrm{pH} \mathrm{CaCl}\left(\mathrm{cmol}_{\mathrm{c}} \mathrm{dm}^{-3}\right)$ & $5,2(0,3) \mathrm{a}$ & $3,1(0,1) \mathrm{b}$ \\
$\mathrm{H}+\mathrm{Al}$ & $5,6(1,5) \mathrm{a}$ & $6,5(1,1) \mathrm{a}$ \\
$\mathrm{Ca}\left(\mathrm{cmol}_{\mathrm{c}} \mathrm{dm}^{-3}\right)$ & $3,7(0,8) \mathrm{a}$ & $0,03(0,04) \mathrm{b}$ \\
$\mathrm{Mg}\left(\mathrm{cmol}_{\mathrm{c}} \mathrm{dm}^{-3}\right)$ & $7,4(1,9) \mathrm{a}$ & $0,13(0,03) \mathrm{b}$ \\
$\mathrm{K}\left(\mathrm{cmol}_{\mathrm{c}} \mathrm{dm}^{-3}\right)$ & $0,9(0,3) \mathrm{a}$ & $0,03(0,01) \mathrm{b}$ \\
$\mathrm{Na}\left(\mathrm{cmol}_{\mathrm{c}} \cdot \mathrm{dm}^{-3}\right)$ & $12,9(3,9) \mathrm{a}$ & $0,04(0,01) \mathrm{b}$ \\
$\mathrm{P}\left(\mathrm{mgdm}^{-3}\right)$ & $9,1(2,2) \mathrm{a}$ & $4,1(0,7) \mathrm{b}$ \\
$\mathrm{C}{\text { orgânico }\left(\mathrm{gdm}^{-3}\right)}_{\mathrm{N} \text { total }\left(\mathrm{gkg}^{-1}\right)}$ & $47,4(7,8) \mathrm{a}$ & $20,6(3) \mathrm{b}$ \\
$\mathrm{CTC}\left(\mathrm{cmol}_{\mathrm{c}} \mathrm{dm}^{-3}\right)$ & $5,7(0,9) \mathrm{a}$ & $0,5(0,1) \mathrm{b}$ \\
$\mathrm{V}(\%)$ & $25,0(6,7) \mathrm{a}$ & $1,0(0,2) \mathrm{b}$ \\
$\mathrm{CE}\left(\mathrm{mScm}^{-1}\right)$ & $79,6(7,5) \mathrm{a}$ & $3,4(0,7) \mathrm{b}$ \\
$\mathrm{Al}\left(\mathrm{cmol}_{\mathrm{c}} \mathrm{dm}^{-3}\right)$ & $9,9(2,3) \mathrm{a}$ & $0,05(0,01) \mathrm{b}$ \\
$\mathrm{Fe}\left(\mathrm{mgkg}^{-1}\right)$ & $0,07(0,10) \mathrm{b}$ & $0,8(0,16) \mathrm{a}$ \\
$\mathrm{Mn}\left(\mathrm{mgkg}^{-1}\right)$ & $67,2(45,2) \mathrm{a}$ & $2,9(0,9) \mathrm{b}$ \\
$\mathrm{Zn}\left(\mathrm{mgkg}^{-1}\right)$ & $58,7(26,2) \mathrm{a}$ & $0,04(0,03 \mathrm{~b}$ \\
$\mathrm{Cu}\left(\mathrm{mgkg}^{-1}\right)$ & $0,9(0,1) \mathrm{a}$ & $0,3(0,06) \mathrm{b}$ \\
\hline
\end{tabular}


si (tabela 2), apesar da diferença dos teores de $\mathrm{Al}$ nos respectivos solos (tabela 1). São escassos os resultados com teores de $\mathrm{Al}$ em indivíduos adultos em florestas naturais. Segundo Geoghegan \& Sprent (1996), os teores de Al encontrados nas folhas do guanandi, em ambos os sítios, indicam que essa espécie é não acumuladora de $\mathrm{Al}$ (valores $<600 \mathrm{mgkg}^{-1}$ ). Possivelmente, o guanandi apresenta mecanismos de tolerância ao $\mathrm{Al}$, a exemplo de outras espécies (Britez et al. 2002). Processos como redução do transporte através da membrana, exclusão, formação de peptídios ricos em grupos tiólicos (fitoquelatinas e metalotioneínas), quelação por ácidos orgânicos e aminoácidos e compartimentalização do metal em estruturas subcelulares permitem a tolerância aos metais pesados pelas plantas (Santos et al. 2006). Plantas excludentes ou acumuladoras de $\mathrm{Al}$ podem coexistir em florestas tropicais, indicando que a concentração do $\mathrm{Al}$ nas plantas é principalmente um processo inerente da espécie do que uma influência das condições edáficas (George et al. 2012). Adicionalmente, a MO pode influenciar na solubilidade do Al, apesar do baixo $\mathrm{pH}$ do solo (Souza et al. 2007).
Apesar de apenas alguns elementos $(\mathrm{K}, \mathrm{Ca}$ e $\mathrm{Cu})$ terem demonstrado diferenças significativas entre as folhas dos sítios estudados, algumas considerações sobre os demais elementos analisados ( $\mathrm{Na}, \mathrm{Fe}$ e $\mathrm{Mn})$ são importantes. Os valores médios de $\mathrm{Na}$ foliar não diferiram estatisticamente entre os indivíduos dos sítios estudados, apesar do solo do Rio Velho, classificado como salino-sódico, apresentar 70 vezes mais $\mathrm{Na}$ do que o solo da Floresta do Palmito, com base na relação $(\mathrm{Na} \%=100 \times \mathrm{Na} / \mathrm{CTC})$. Os teores de Na na Floresta do Palmito podem ser explicados pelo aporte do $\mathrm{Na}$ via spray marinho e, nas folhas, pela competição iônica do $\mathrm{Na}$ com os teores de $\mathrm{Ca}, \mathrm{Mg}$ e/ ou K, que se encontram em baixa concentração neste solo (tabela 1). Uma vez que o guanandi não apresenta estruturas secretoras de sal nas suas folhas (Boeger \& Wisniewski 2003), a espécie pode ser classificada como tolerante à salinidade (EMBRAPA 1999), de acordo com os dados da CE (tabela 1).

Tanto o Fe como o Mn não apresentaram relação direta dos teores foliares com os teores encontrados no solo. O Fe apresentou relação inversa, onde teores de Fe são inversamente proporcionais entre as folhas e o

Tabela 2. Médias e respectivos desvios-padrão dos teores foliares de macronutrientes $\left(\mathrm{g} \mathrm{kg}^{-1}\right)$, sódio $\left(\mathrm{Na}, \mathrm{g} \mathrm{kg}^{-1}\right)$, micronutrientes $\left(\mathrm{mgkg}^{-1}\right)$, alumínio $\left(\mathrm{Al}, \mathrm{mg} \mathrm{kg}^{-1}\right)$, área específica foliar $\left(\mathrm{AEF}, \mathrm{cm}^{2} \mathrm{~g}^{-1}\right)$ e razões $\mathrm{C} / \mathrm{N}, \mathrm{C} / \mathrm{P}$ e N/P de Calophyllum brasiliense nos sítios de coleta. Letras diferentes, em linha, representam diferenças significativas, teste de Duncan, $\alpha=5 \%$.

Table 2. Mean values and respective standard deviations of leaf macronutrient $\left(\mathrm{g} \mathrm{kg}^{-1}\right)$, sodium $\left(\mathrm{Na}, \mathrm{g} \mathrm{kg}^{-1}\right)$, micronutrients ( $\left.\mathrm{mg} \mathrm{kg}^{-1}\right)$, aluminum (Al, $\left.\mathrm{mg} \mathrm{kg}^{-1}\right)$ contents, leaf specific area $\left(\mathrm{AEF}, \mathrm{cm}^{2} \mathrm{~g}^{-1}\right)$ and $\mathrm{C} / \mathrm{N}, \mathrm{C} / \mathrm{P}$ and N/P ratios of Calophyllum brasiliense in the studied sites. Different letters on the line represent significant differences, Duncan test, $\alpha=5 \%$.

\begin{tabular}{lcc}
\hline & \multicolumn{2}{c}{ Sítios } \\
\cline { 2 - 3 } & Rio Velho & Floresta do Palmito \\
\hline $\mathrm{C} \%$ & $46,4(1,7) \mathrm{a}$ & $47,5(0,4) \mathrm{a}$ \\
$\mathrm{N}$ & $10,7(1,2) \mathrm{a}$ & $12,3(0,7) \mathrm{a}$ \\
$\mathrm{C} / \mathrm{N}$ & $43,6(3,3) \mathrm{a}$ & $38,7(2,5) \mathrm{a}$ \\
$\mathrm{C} / \mathrm{P}$ & $591,5(18,4) \mathrm{a}$ & $598,5(46,6) \mathrm{a}$ \\
$\mathrm{N} / \mathrm{P}$ & $13,7(0,8) \mathrm{a}$ & $15,8(0,2) \mathrm{a}$ \\
$\mathrm{P}$ & $0,8(0,03) \mathrm{a}$ & $0,8(0,07) \mathrm{a}$ \\
$\mathrm{K}$ & $6,3(0,5) \mathrm{a}$ & $4,2(0,6) \mathrm{b}$ \\
$\mathrm{Ca}$ & $5,20,9) \mathrm{a}$ & $2,7(0,5) \mathrm{b}$ \\
$\mathrm{Mg}$ & $2,0(0,4) \mathrm{a}$ & $2,7(0,4) \mathrm{a}$ \\
$\mathrm{Na}$ & $0,6(0,1) \mathrm{a}$ & $0,6(0,3) \mathrm{a}$ \\
$\mathrm{Fe}$ & $34,1(13,3) \mathrm{a}$ & $48,9(14,3) \mathrm{a}$ \\
$\mathrm{Mn}$ & $13,4(4,0) \mathrm{a}$ & $13,6(2,3) \mathrm{a}$ \\
$\mathrm{Zn}$ & $13,1(0,8) \mathrm{a}$ & $13,0(0,7) \mathrm{a}$ \\
$\mathrm{Cu}$ & $3,0(1,5) \mathrm{b}$ & $6,1(3,7) \mathrm{a}$ \\
$\mathrm{Al}$ & $127,0(4,8) \mathrm{a}$ & $159,6(20,6) \mathrm{a}$ \\
$\mathrm{AFE}$ & $55,79( \pm 3,0) \mathrm{b}$ & $61,56( \pm 8,0) \mathrm{a}$ \\
\hline
\end{tabular}


solo (tabela 1). Para o Mn, os teores no solo no sítio Floresta do Palmito são muito baixos enquanto que nas folhas, os teores são semelhantes aos encontrados para nos indivíduos do sítio do Rio Velho (tabela 1). Os resultados sugerem a importância do aporte da matéria orgânica na ciclagem desses e de outros elementos, principalmente no sítio da Floresta do Palmito, onde os valores médios de alguns elementos (ex. $\mathrm{Mn}$ e Ca) no solo foram praticamente ausentes.

Os valores médios de $\mathrm{P}, \mathrm{K}, \mathrm{Na}, \mathrm{Fe}$ e $\mathrm{Al}$ foram maiores e significantes na serapilheira no Rio Velho, enquanto que apenas os valores médios de Mn foram maiores na serapilheira na Floresta do Palmito (tabela 3). Apesar dos maiores valores de Al na serapilheira do Rio Velho, é importante ressaltar que os valores desse elemento também podem ser considerados altos (tabela 3) na serapilheira da Floresta do Palmito (Britez et al. 2002). Os baixos valores médios de Al nos solos do Rio Velho e da Floresta do Palmito (tabela 1) indicaram a ocorrência da translocação do elemento para as folhas senescentes.

Para os dois sítios (tabela 3), é evidente que a fertilidade é dependente da MO acumulada nos horizontes orgânicos. Especialmente na Floresta do Palmito, a presença do acúmulo de raízes ativas nas camadas superficiais dos horizontes orgânicos indica a importância desse horizonte no processo de ciclagem de nutrientes (Boeger et al. 2005). Além disso, estudos adicionais sobre os horizontes orgânicos na Floresta do Palmito mostraram que a contribuição de $\mathrm{Mn}$ e Ca pela MO correspondem a 288,67 mg kg-1 e 7,44 $\mathrm{g} \mathrm{kg}^{-1}$, respectivamente (Protil et al. 2006).

Apesar dos menores valores de área específica foliar ocorrerem nas folhas do Rio Velho (tabela 2), as folhas dos indivíduos dos dois sítios são consideradas esclerófilas. A esclerofilia é definida como uma resposta não específica a ambientes com múltiplos estresses ambientais (Read et al. 2006). Plantas esclerófilas desenvolvem folhas resistentes à herbivoria, seca, baixa fertilidade do solo (devido ao baixo Teor de N e P) e/ou alta salinidade (Feller 1996, Boeger \& Wisniewski 2003, Hanley et al. 2007), como uma medida conservativa de nutrientes e/ou massa verde (Turner 1994).

A esclerofilia no guanandi resultou do maior investimento estrutural principalmente nos tecidos foliares com parede secundária, resultante das altas relações $\mathrm{C} / \mathrm{N}$ e $\mathrm{C} / \mathrm{P}$ nas folhas, em ambos os sítios. Tanto o $\mathrm{N}$ com o $\mathrm{P}$ apresentaram valores muito baixos nas folhas, como indicado pela relação N/P (tabela 2). Valores de N/P entre 14 e 16 indicam que tanto o $\mathrm{N}$ e o P podem ser limitantes (Koerselman \&

Tabela 3. Médias e respectivos desvios-padrão dos macronutrientes $\left(\mathrm{g} \mathrm{kg}^{-1}\right)$, micronutrientes $\left(\mathrm{mg} \mathrm{kg}^{-1}\right), \mathrm{Sódio}\left(\mathrm{Na}, \mathrm{g} \mathrm{kg}^{-1}\right)$ e Alumínio (Al, $\mathrm{mg} \mathrm{kg}^{-1}$ ) da serapilheira acumulada nos sítios de coleta. Letras diferentes, em linha, representam diferenças significativas, teste de Duncan, $\alpha=5 \%$.

Table 3. Mean values and respective standard deviations of macronutrients $\left(\mathrm{gkg}^{-1}\right)$, micronutrients $\left(\mathrm{mgkg}^{-1}\right), \mathrm{Sodium}(\mathrm{Na}$, $\left.\mathrm{gkg}^{-1}\right)$, and Aluminum (Al, $\mathrm{mgkg}^{-1}$ ) of accumulated litter in the study sites. Different letters on the line represent significant differences, Duncan test, $\alpha=5 \%$.

\begin{tabular}{lcc}
\hline & \multicolumn{2}{c}{ Sítios } \\
\cline { 2 - 3 } $\mathrm{C}$ & Rio Velho & Palmito \\
$\mathrm{N}$ & $47,6(0,8) \mathrm{a}$ & $48,1(0,8) \mathrm{a}$ \\
$\mathrm{C}: \mathrm{N}$ & $8,2(1,1) \mathrm{a}$ & $9,6(2,1) \mathrm{a}$ \\
$\mathrm{P}$ & $58,4(7,6) \mathrm{a}$ & $52,1(10,3) \mathrm{a}$ \\
$\mathrm{K}$ & $0,92(0,15) \mathrm{a}$ & $0,61(0,06) \mathrm{b}$ \\
$\mathrm{Ca}$ & $3,3(0,4) \mathrm{a}$ & $1,8(0,5) \mathrm{b}$ \\
$\mathrm{Mg}$ & $7,0(1,5) \mathrm{a}$ & $5,2(1,2) \mathrm{a}$ \\
$\mathrm{Na}$ & $2,4(0,4) \mathrm{a}$ & $2,4(0,4) \mathrm{a}$ \\
$\mathrm{Fe}$ & $1,7(0,7) \mathrm{a}$ & $0,3(0,08) \mathrm{b}$ \\
$\mathrm{Mn}$ & $200,5(114,9) \mathrm{a}$ & $63,6(14,1) \mathrm{b}$ \\
$\mathrm{Zn}$ & $17,2(4,4) \mathrm{b}$ & $34,8(7,6) \mathrm{a}$ \\
$\mathrm{Cu}$ & $15,5(2,7) \mathrm{a}$ & $15,7(2,9) \mathrm{a}$ \\
$\mathrm{Al}$ & $5,7(1,3) \mathrm{a}$ & $6,3(1,6) \mathrm{a}$ \\
\hline
\end{tabular}


Meuleman 1996). Assim, o C excedente pode estar sendo utilizado na formação das paredes celulares secundárias dos tecidos mecânicos e de transporte (xilema). Esse investimento em $\mathrm{C}$ na estrutura da folha representa um importante caráter ecológico, tornando as folhas mais duras e com maior proteção à perda de nutrientes (Lambers et al. 1998). O índice de esclerofilia encontrados nas folhas nos dois sítios é resposta a condição de estresse ao baixo teor de $\mathrm{N}$ em ambos os sítios, além do alto teor de $\mathrm{Al}$ e a baixa fertilidade do solo na Floresta do Palmito e à alta salinidade do solo no Rio Velho.

\section{Agradecimentos}

À CAPES, pela concessão da bolsa a primeira autora. Ao Dr. Ricardo M. de Britez e funcionários da SPVS, ao Instituto Ambiental do Paraná (IAP) e ao Leandro José da Silva pelo auxílio nas coletas, ao Dr. Edílson B. de Oliveira pelo auxílio nas análises estatísticas e ao CNPq pela concessão da bolsa de produtividade a MRT Boeger.

\section{Literatura citada}

Barber, S.A. 1995. Soil Nutrient Bioavailability: a mechanistic approach. John Wiley, New York.

Boeger, M.R.T. \& Wisniewski, C. 2003 Comparação da morfologia foliar de espécies arbóreas de três estádios sucessionais distintos de floresta ombrófila densa (Floresta Atlântica) no Sul do Brasil. Revista Brasileira de Botânica 26: 61-72.

Boeger, M.R.T., Wisniewski, C. \& Reissmann, C.B. 2005. Nutrientes foliares de espécies arbóreas de três estádios sucessionais de floresta ombrófila densa no sul do Brasil. Acta Botanica Brasilica 19: 167-181.

Bonilha, R.M. 2012. Caracterização da fertilidade do solo, distribuição do sistema radicular e índice de qualidade do solo no ecossistema restinga do Litoral Paulista. Dissertação de Mestrado em Agricultura e Ambiente. Universidade Federal de São Carlos. São Carlos, SP.

Bremner, J.M. 1996. Nitrogen-total. In: Soil Science Society of America Book Series. Methods of Soil Analysis. Madison: Soil Science Society of America Book Series.

Britez, R.M., Watanabe, T., Jasen, S., Reissmann, C.B. \& Osaki, M. 2002. The relationship between aluminum and silicon accumulation in leaves of Faramea marginata (Rubiaceae). New Phytologist 156: 437-444.

Carvalho, P.E.R. 2003. Espécies arbóreas brasileiras. v. 1. EMBRAPA, Brasília.

EMBRAPA - Empresa Brasileira de Pesquisa Agropecuária. 1999. Sistema Brasileiro de Classificação de Solos. EMBRAPA, Rio de Janeiro.
Feller, I.C. 1996. Effects of nutrient enrichment on leaf anatomy of dwarf Rhizophora mangle L. (red mangrove). Biotropica 28: 13-22.

Geoghegan, I.E. \& Sprent, J.I. 1996. Aluminium and nutrient concentration in species native to Central Brazil. Communications in Soil Science Plant Analysis 27: 2925-2934.

George, W.J.H. \& Neumann, E. 2012. Adaptation of plants to adverse chemical soil conditions. In: P. Marschner (ed.). Marchner's mineral nutrition of higher plants.: Academic Press, Waltham.

Hanley, M.E., Lamont, B.B., Fairbanks, M.M. \& Rafferty, C.M. 2007. Plant structural traits and their role in anti-herbivore defence. Perspectives in Plant Ecology, Evolution and Systematics 8: 157-178.

Jordão, C.P., Alves, N.M., Pereira, J.L. \& Bellato, C.R. 2000. Adsorção de íons $\mathrm{Cu}^{+2}$ em Latossolo vermelhoamarelo húmico. Química Nova 23: 5-11.

Koerselman, W. \& Meuleman, A.F.M. 1996. The vegetation N:P ratio: a new tool to detect the nature of nutrient limitation. Journal of Applied Ecology 33: 1441-1450.

Lambers, H., Chapin III F.S. \& Pons, T.L. 1998. Plant physiological ecology. Springer, New York, NY.

Luchese, E.B., Favaro, L.O.B. \& Lenzi, E. 2001. Fundamentos da química do solo. Freitas Bastos Editora, Rio de Janeiro.

Martins, A.P.L. \& Reissmann, C.B. 2007. Material vegetal e as rotinas laboratoriais nos procedimentos químicoanalíticos. Scientia Agraria 8: 1-17.

Mengel, K \& Kirkby, E.A. 1987. Principles of Plant Nutrition. Bern: International Potash Institute, Bern.

Meurer, E.J. 2006. Fundamentos de química do solo. 3 ed. Evangraf, Porto Alegre, RS.

Ndaw, S.M., Gama-Rodrigues, A.C., Gama-Rodrigues, E.F., Sales, K. R.N. \& Rosado, A.S. 2009. Relationships between bacterial diversity, microbial biomass, and litter quality in soils under different plant covers in northern Rio de Janeiro State, Brazil. Canadian Journal of Microbiology 55: 1089-1095.

Pavan, M.A., Bloch, M.F., Zempulski, H.C., Miyazawa, M. \& Zocoler, D.C. 1992. Manual de análise química de solo e controle de qualidade. Circular IAPAR 76. Instituto Agronômico do Paraná, Londrina.

Prescott, C.E. 2002. The influence of the forest canopy on nutrient cycling. Tree Physiology 22: 1193-1200.

Protil, C.Z., Marques, R \&Protil, R.M. 2009. Variação sazonal e redistribuição de biolementos de quatro espécies arbóreas em três tipologias florestais da Floresta Atlântica do Paraná. Floresta 39: 699-717.

Raij, B.V., Gheyi, H.R. \& Bataglia, O.C. 2001. Determinação da condutividade elétrica e de cátions solúveis em extratos aquosos de solos. In: B.V. Raij, J.C. Andrade, H. Cantarella \& J.A. Quaggio, (eds.). Análise química para Avaliação da fertilidade de solos tropicais. Instituto Agronômico, Campinas, SP. 
Read, J., Sanson, G.D., Garine-Wichatitsky, M. \& Jaffré, T. 2006. Sclerophylly in two contrasting tropical environments: low nutrients vs. low rainfall. American Journal of Botany 93: 1601-1604.

RieuwertS, J.S. 2007. The mobility and bioavailability of trace metals in tropical soils: a review. Chemical Speciation and Bioavailability 19: 75-85.

Santo Jr., C.E. 2008. Atlas geográfico do Paraná. Secretaria de Educação do Estado do Paraná. SEED/PR, Curitiba. PR.

Santos, F.S., Amaral Sobrinho, N.M.B. \& Mazur, N. 2006. Mecanismos de tolerância de plantas e metais pesados. In: M.S. Fernandes (ed.). Nutrição mineral de Plantas. Sociedade Brasileira do Solo, Viçosa, MG.

Silva, F.C. 1999. Manual de análises químicas de solo, plantas e fertilizantes. Embrapa, Brasília.
Silveira, M.L.A. \& Alleoni, L.R.F. 2003. Copper adsorption in tropical oxisols. Brazilian Archives of Biology and Technology 46: 529-536.

SPVS - Sociedade de Pesquisa em Vida Selvagem. 2002. Levantamento de Solos: Reserva Natural Serra do Itaqui. Relatório Técnico, Curitiba.

Souza, D.M.G., Miranda, L.N. \& Oliveira, S.A. 2007. Acidez do solo e sua correção. In: R.F. Novais, V.H. Alvarez, N.F. de Barros, R.L.F. Fontes, B. Cantarutti \& J.C.L. Neves (eds.). Fertilidade do Solo. Sociedade Brasileira de Ciência do Solo, Viçosa, MG.

Turner, I.M. 1994. A quantitative analysis of leaf form in woody plants from the world's major brodleaved forest types. Journal of Biogeography 21: 413-419.

Zacarias, R.R., Britez, R.M., Galvão, F., Boeger, M.R.T. 2012. Fitossociologia de dois trechos de floresta ombrófila densa aluvial em solos hidromórficos, Paraná, Brasil. Floresta 42: 769-782. 